\title{
Analysis and Simulation of Multi-hop Connection Probability of Non-independent VANETs
}

\author{
Zhang Tiandan and Cao Li \\ 1. Department of Information Technology, Nantong Normal College, Jiangsu \\ Nantong, 226000, China \\ 2. School of Computer Science and Technology, Nantong University, Jiangsu \\ Nantong, 226000, China
}

\begin{abstract}
For the connection problems of VANETs, the paper analyzes the link connection model. Existing studies on multi-hop connection assume that the link is independent and identically distributed and did not consider the non-independence between continuous links. For this purpose, this paper presents a multi-hop connection probability model based on non independent links. By the relative distance between vehicles, combined with the vehicle movement model, the vehicle distribution is derived and the probability of multi-hop connection is deduced. The simulation data show that the connection probability varies with the relative distance of the vehicles and the probability of the connection decreases with the increase of relative distance, consistent with the results of the theoretical derivation.
\end{abstract}

Keywords: Connection Probability, Multi-Hop, Moving Model, Vanets (Vehicular Ad Hoc Networks)

\section{Introduction}

In recent years, VANETs (Vehicular ad hoc networks) has been widely concerned. There are two main facts: first, a large number of traffic accidents annually, and second, proposal of vehicle communication standards, such as IEEE802.11p and Dedicated Short Range Communications (DSRC). The purpose of research on the VANETs is to realize the interaction of the security and emergency information of moving vehicles. By installing the global positioning system GPS and other sensing devices, the vehicles can acquire movement information, such as the current position, speed, moving direction and the like. The broadcast is often used to share the vehicle moving information within hop region. The mobile information obtained from the neighbor vehicles plays an important role in the security early warning and routing decision. A large number of research works have emerged for the multi hop connection of VANETs. The purpose of the study is to improve the probability of multi hop connection. As is well known, the connection probability of multi hop VANETs is influenced not only by the radius of the vehicle communication, but also by the distance between the moving vehicles. In literature [3], the authors assume that through the whole communication phase, all forwarding vehicles should be within a hop communication range. In literature [4], the establishment of multi hop paths between source and destination nodes is defined as: the distance between each vehicle is independent and identically distributed, and the vehicle-vehicle distance is less than or equal to the vehicle communication radius. In literature [5] and [6], the authors use a similar method to obtain the multi-hop path duration from the minimum duration of each link. In addition, these two documents assume that multiple links are independent of each other. However, as far as I know, a large number of existing literatures did not consider the multi-hop non-independence as well as the influence of each other. Literature [7] describes part of the simulation result. Based on vehicle spacing independence, the 
establishment of a multi-hop path is simply defined as the link probability of single links. To this end, the paper presents a new approach to analyze multi-hop connection model. This model combines the traffic flow information and the relative distance between vehicles. The multi-hop connectivity is described by the probability of the vehicle appearing in the forwarding area. At the same time, it confirms that the probability of multi-hop connection obeys the exponential distribution, and changes with the arrival rate of vehicles.

\section{Moving Model}

Since the expressway is one of the most common scenarios with only two moving directions, the moving model established in this paper is based on the expressway scene. As the vehicle communication radius is much larger than the width of the road, the scene to be analyzed can be simplified to a one-dimensional expressway scene.

Vehicle moving model discussed in this section refers to the thoughts in literature [3, 8]. In this model, the vehicle with the arrival rate of $\beta$ per second obeying Poisson distribution appears at a certain position of the expressway. Therefore, the arrival probability of $k$ nodes (vehicles) in the time domain $[0, t]$ is:

$$
\operatorname{Pr}(N(t)=k)=\frac{(\beta t)^{k} e^{-\beta t}}{k !}
$$

The moving route of each vehicle is composed of a series of random vehicle intervals, which obey e exponential distribution with the mean value of $1 / \alpha$. The length of interval $i$ is $\left\{T_{i}: i \geq 1\right\}$. Therefore, the number of intervals in time domain of $[0, t]$ can be expressed as:

$$
t=\sum_{i=1}^{M(t)} T_{i}
$$

$M(t)$ is the counting process of exponential distribution. Therefore, $M(t)$ obeys Poisson distribution of $\alpha t$.

The vehicle starts at each interval to travel at a randomly selected speed from $\left[v_{\min }, v_{\max }\right]$ and maintains a uniform motion. Thus, the speed of the vehicle is subject to the same mean value of $\mu=\frac{v_{\max }+v_{\min }}{2}$, the variance of $\delta^{2}=\frac{\left(v_{\max }-v_{\min }\right)^{2}}{12}$, uniformly distributed.

For a particular vehicle $M$, with the moving speed of $v_{i, M}$ at interval $i$ for $T_{i, M}$, the distance travelled can be expressed as $D_{i, M}=v_{i, M} \times T_{i, M}$. As the vehicle moves independently of each other, the moving model obeys the same distribution. Thus, the subscript can be omitted, i.e. $D_{i}=v_{i} \times T_{i}$. Throughout the time domain $[0, t]$, the entire distance $D(t)$ of a vehicle moving can be express as:

$$
D(t)=\sum_{i=1}^{M(t)} D_{i}=\sum_{i=1}^{M(t)} v_{i} \times T_{i}
$$

Since $\left\{D_{i}: i \geq 1\right\}$ is an independent and identically distributed random variable, and $M(t)$ is the Poisson process and independent of $D_{i},\{D(t): t \geq 0\}$ obeys complex Poisson 
process. Its mean value $E[D(t)]$ and variance $\operatorname{var}[D(t)]$ can be expressed respectively as:

$$
\begin{gathered}
E[D(t)]=\alpha t E\left[D_{i}\right]=\mu t \\
\operatorname{var}[D(t)]=\alpha t E\left[D_{i}^{2}\right]=\frac{2 t}{\alpha}\left(\mu^{2}+\delta^{2}\right)
\end{gathered}
$$

According to the central limit theorem, it can be approximated that $D(t)$ obeys the normal distribution of the same mean value $E[D(t)]$ and variance $\operatorname{var}[D(t)]$. The moving state of any vehicle can be described by the entry time of the reference vehicle ${ }^{t}$ and the time difference $\tau$ between the reference vehicle and the observation vehicle. Assuming $\tau>0$, it represents that the observation vehicle joins the system before the reference vehicle, on the contrary, behind. Therefore, $D(t+\tau)$ represents the whole distance the observation vehicle travels in the time domain $[0, t+\tau]$, obeying the normal distribution of the mean value $E[D(t+\tau)]$ and variance $\operatorname{var}[D(t+\tau)]$, which can be respectively expressed as:

$$
\begin{array}{r}
E[D(t+\tau)]=\mu(t+\tau)(6) \\
\operatorname{var}[D(t+\tau)]=\frac{2(t+\tau)}{\alpha}\left(\mu^{2}+\delta^{2}\right)
\end{array}
$$

The relative distance between the observation vehicle and the reference vehicle can be observed by the difference of their moving distance, such as $D_{r}(\tau, t)=D(t+\tau)-D(t)$, and its mean value $E\left[D_{r}\right]$ and variance $\operatorname{var}\left[D_{r}\right]$ can be expressed as:

$$
\begin{gathered}
E\left[D_{r}\right]=\mu \tau=\mu_{r} \\
\operatorname{var}\left[D_{r}\right]=\frac{2(2 t+\tau)}{\alpha}\left(\mu^{2}+\delta^{2}\right)=\delta_{r}^{2}
\end{gathered}
$$

Therefore, $\left\{D_{r}(\tau, t):-\frac{t}{2} \leq \tau \leq \frac{t}{2}, t \geq 0\right\}$ can be approximated to obey normal distribution, with the mean value of $\mu_{r}$ and the variance of $\delta_{r}^{2}$.

$$
f_{D_{r}}(x)=\frac{1}{\sqrt{2 \pi \delta_{r}^{2}}} \exp \left(-\frac{\left(x-\mu_{r}\right)^{2}}{2 \delta_{r}^{2}}\right)
$$

\section{Performance Analysis}

Assume that all the vehicles have the same receiver and transmitter. If the distance between vehicles is less than or equal to the communication radius, the link between them will be established. Therefore, as long as the vehicles are within the communication range, they will be able to transmit information directly. On the contrary, if the vehicles are out of the range of communication, they need to forward messages with the intermediate node.

\subsection{Two-Hop Connection Probability during the Establishment of Connections}


As is shown in Figure 1, the source node $\mathrm{C}$ and destination node $\mathrm{A}$ move to the right along the road. When the distance between $A B$ and $B C$ is less than $R$, node $B$, as the forwarding node of node $\mathrm{A}$ and $\mathrm{C}$, realizes the communication between $\mathrm{A}$ and $\mathrm{C}$. The shadow part of the diagram indicates the forwarding area of node B. Thus, the next is analysis of the probability of forwarding node B appearing in the forwarding area.

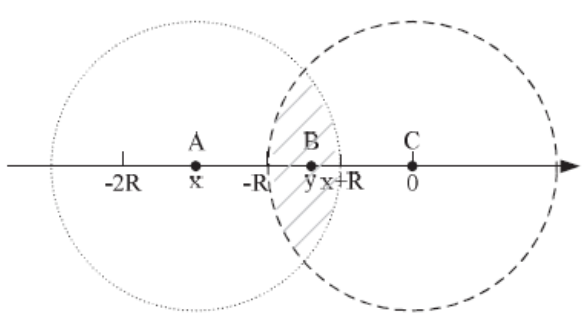

\section{Figure 1. Two-Hop Indirect Communication during the Establishment of}

\section{Links}

Assuming the reference vehicle is the source node $\mathrm{C}$, the time it enters the system is 0 . At time $t$, node $\mathrm{C}$ needs to establish a communication path with the destination node $\mathrm{A}$. At this time, the position of node $\mathrm{A}$ is $x,-2 R \leq x \leq 2 R$. However, they cannot directly establish a communication path, but if there is a node in the shadow area $[-R, x+R]$, there will be a potential indirect connection between node $\mathrm{A}$ and $\mathrm{C}$. Thus, a particular observation vehicle, with time difference $\tau$ from the reference vehicle, appears in the region $[-R, x+R]$, and then, based on formula (10), the probability of establishing connection at time $t$ is:

$$
P_{\tau 1}(x)=\int_{-R}^{x+R} f_{D_{r}}(y) d y
$$

Because it is a Poisson process, the vehicle arrival time in time domain $\left(-\frac{t}{2}, \frac{t}{2}\right)$ obeys uniform distribution. When the time difference exceeds the range $\left(-\frac{t}{2}, \frac{t}{2}\right)$, the probability of the vehicle appears in the area $[-R, x+R]$ at time ${ }^{t}$ is too small to establish a connection. Therefore, if there are $n$ vehicles entering the system within $\left(-\frac{t}{2}, \frac{t}{2}\right)$, the probability of any vehicle appearing in $[-R, x+R]$ is:

$$
\begin{aligned}
P_{t 1}(x) & =\frac{1}{t} \int_{-\frac{t}{2}}^{\frac{t}{2}} P_{\tau 1}(x) d \tau \\
& =\frac{1}{t} \int_{-\frac{t}{2}}^{\frac{t}{2}} \int_{-R}^{x+R} \frac{1}{\sqrt{2 \pi \delta_{r}^{2}}} \exp \left(-\frac{\left(y-\mu_{r}\right)^{2}}{2 \delta_{r}^{2}}\right) d y d \tau
\end{aligned}
$$

Therefore, the probability of any vehicle being out of the area $[-R, x+R]$ is $1-P_{t 1}(x)$.

Assume that $n$ vehicles enter the system with the time difference of $\tau$ from the reference vehicle, $\left\{\tau:-\frac{t}{2}<\tau<\frac{t}{2}\right\}$. Among the $n$ vehicles, $N_{1}(t)$ vehicles appear in the area $[-R, x+R]$ at time ${ }^{t}$. The prerequisite of connecting the initial position 0 to $x$ is that 
there is at least one intermediate node within $[-R, x+R]$. Therefore, the connection probability $P_{c 1}^{t}(x)$ from position ${ }^{x}$ to the initial position 0 can be expressed as:

$$
P_{c 1}^{t}(x)=1-P_{0}^{t}(x)
$$

In this formula, $P_{0}^{t}(x)$ indicates that there is no vehicle in the area $[-R, x+R]$. According to the preceding analysis, vehicles obey the Poisson process of arrival rate $\beta$. Thus, $P_{0}^{t}(x)$ can be expressed as:

$$
\begin{aligned}
P_{0}^{t}(x) & =\sum_{n=0}^{\infty}\left[1-P_{t 1}(x)\right]^{n} \frac{1}{n !} \exp \left(-\beta t(\beta t)^{n}\right) \\
& =\exp \left(-\beta t P_{t 1}(x)\right)
\end{aligned}
$$

Accordingly, at position $x(-2 R \leq x \leq 2 R)$, the two-hop connection probability $P_{c 1}^{t}(x)$ can be expressed as:

$$
P_{c 1}^{t}(x)=1-\exp \left(-\beta t P_{t 1}(x)\right)
$$

\subsection{Two-Hop Connection Probability during the Breaking Link Period}

As is shown in Figure 2, the destination node A may move out of the communication range of intermediate node $\mathrm{B}$. When the intermediate node $\mathrm{B}$ leaves the area $[x-R, R]$, the indirect link between node A and reference node $C$ will break. Similar to section 3.1, the probability $P_{\tau 2}(x)$ of a node appearing in position ${ }^{x}$ with the time difference of ${ }^{\tau}$ and the probability $P_{t 2}(x)$ of any vehicle appearing in the area $[x-R, R]$ can be respectively expressed as:

$$
\begin{aligned}
P_{\tau 1} & (x)=\int_{x-R}^{R} \frac{1}{\sqrt{2 \pi \delta_{r}^{2}}} \exp \left(-\frac{\left(y-\mu_{r}\right)}{2 \delta_{r}^{2}}\right) d y(16) \\
P_{t 2}(x) & =\frac{1}{t} \int_{-\frac{t}{2}}^{\frac{t}{2}} P_{\tau 2}(x) d \tau \\
& =\frac{1}{t} \int_{-\frac{t}{2}}^{\frac{t}{2}} \int_{x-R}^{R} \frac{1}{\sqrt{2 \pi \delta_{r}^{2}}} \exp \left(-\frac{\left(y-\mu_{r}\right)^{2}}{2 \delta_{r}^{2}}\right) d y d \tau
\end{aligned}
$$

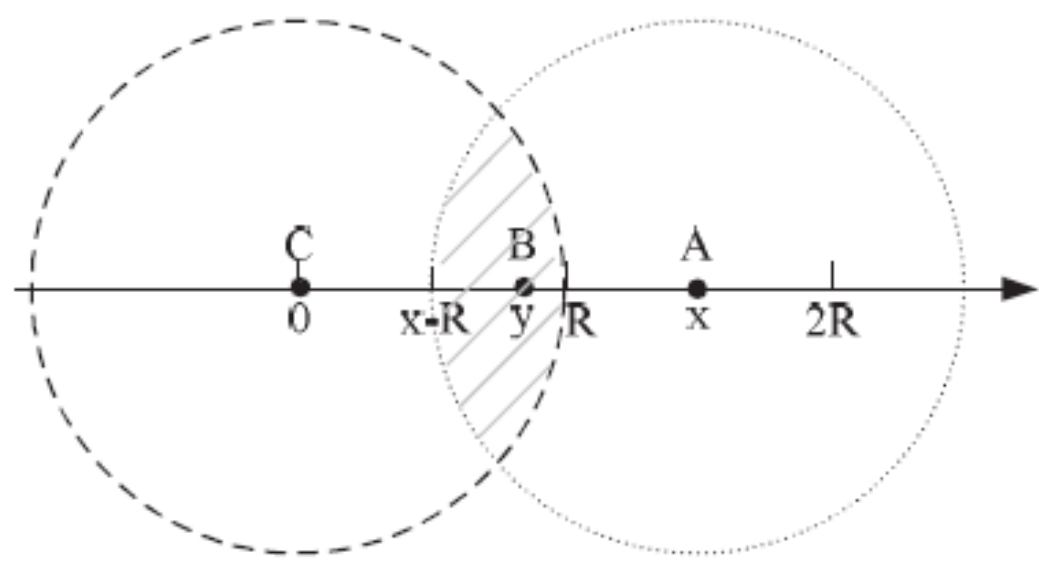




\section{Figure 2. Two-Hop Indirect Communications during the Breaking Link Period}

Similarly, $N_{2}(t)$ represents the number of nodes in the area $[x-R, R]$. At position $x($ $R<x \leq 2 R$ ), the two-hop connection probability can be expressed as:

$$
P_{c 2}^{t}(x)=1-\exp \left(-\beta t P_{t 2}(x)\right)
$$

\subsection{Multi-hop Connection Probability}

There are two basic situations for vehicles switching from two-hop indirect communication to multi-hop mode, as are shown in Figure 3 and Figure 4. In Figure 3, the distance between the destination node $\mathrm{A}$ and the source node $\mathrm{C}$ is $x$, and $-3 R<x \leq-2 R$. If there is an intermediate node $\mathrm{B}_{1}$ located in $\left[x_{2}-R, x+R\right]$, and another intermediate node $\mathrm{B}_{2}$ located in $\left[-R, x_{1}+R\right]$, a link can be established between node $\mathrm{A}$ and $\mathrm{C}$, where $x_{1}$ and $x_{2}$ represent the position of the two intermediate nodes respectively, and $x_{1}<x_{2}$.

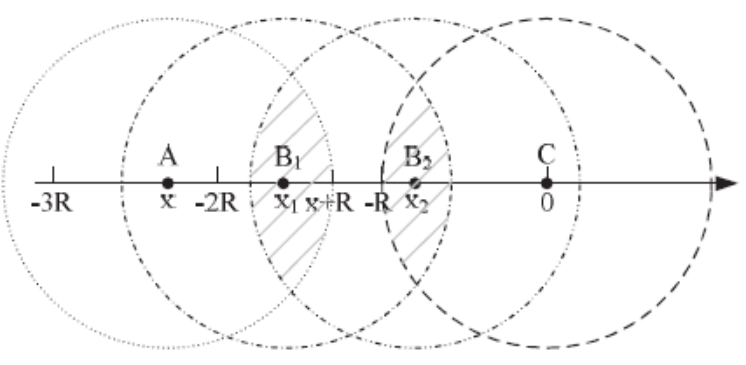

Figure 3. Three-Hop Indirect Communication: Situation 1

In Figure 4, there are no intermediate nodes within the area $[-R, x+R]$, which results in the failure to establish a two-hop connection between node $\mathrm{A}$ and node $\mathrm{C}$. If the intermediate nodes $\mathrm{B}_{1}$ and $\mathrm{B}_{2}$ can appear in the areas $\left[x_{2}-R,-R\right]$ and $\left[x+R, x_{1}+R\right]$, the connection between node $\mathrm{A}$ and node $\mathrm{C}$ can be established by three-hop mode. Because intermediate node $\mathrm{B}_{1}$ and $\mathrm{B}_{2}$ can communicate directly with the vehicle $\mathrm{A}$ and $\mathrm{C}$, the distance between $B_{1}$ and $B_{2}$ is less than the communication radius $R$.

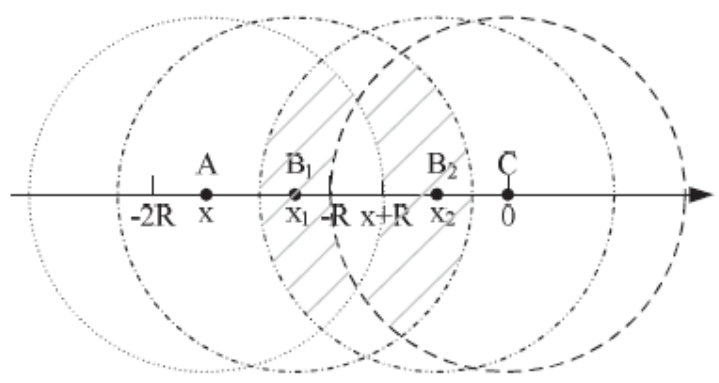

Figure 4. Three-Hop Indirect Communication: Situation 2

For the two situations in Figure 3 and Figure 4, restrictions on the area where intermediate nodes appear can be simplified, as are shown in formula (19) and (20): 


$$
\begin{aligned}
& \left\{\begin{array}{c}
x_{2}-R \leq x_{1} \leq x+R \\
-R \leq x_{2} \leq x+2 R
\end{array}\right. \\
& \left\{\begin{array}{c}
x_{2}-R \leq x_{1} \leq x-R \\
x+R \leq x_{2} \leq 0
\end{array}\right.
\end{aligned}
$$

Therefore, in Figure 3, the probability $P_{t 3}(x)$ of any two random nodes appearing respectively in the area $[-R, x+2 R]$ and $\left[x_{2}-R, x+R\right]$ can be expressed as formula (21) and (22):

$P_{t 3}(x)=\frac{1}{t} \int_{-\frac{t}{2}}^{\frac{t}{2}} \int_{-R}^{x+2 R} \int_{x_{2}-R}^{x+R} f_{D_{r}}\left(x_{1}\right) f_{D_{r}}\left(x_{2}\right) d x_{1} d x_{2} d \tau$

Similarly, the probability $P_{t 4}(x)$ in Figure 4 can be expressed as:

$P_{t 4}(x)=\frac{1}{t} \int_{-\frac{t}{2}}^{\frac{t}{2}} \int_{x+R}^{0} \int_{x_{2}-R}^{-R} f_{D_{r}}\left(x_{1}\right) f_{D_{r}}\left(x_{2}\right) d x_{1} d x_{2} d \tau$

Therefore, the three-hop probability $P_{c 3}^{t}(x)$ and $P_{c 4}^{t}(x)$ for the two areas $-3 R \leq x \leq-2 R$ and $-2 R \leq x \leq-R$ can be expressed as:

$$
\begin{aligned}
& P_{c 3}^{t}(x)=1-\exp \left(-\beta t P_{t 3}(x)\right) \\
& P_{c 4}^{t}(x)=1-\exp \left(-\beta t P_{t 4}(x)\right)
\end{aligned}
$$

\section{Performance Analysis}

In this section, MATLAB software is used to simulate and analyze the performance of the system. In order to confirm the correctness of the proposed model and the derivation and analysis, a one-way expressway scene with two-hop communication is established and the theoretical derivation of the two-hop connection probability is compared with the experimental simulation of the expressway.

Table 1. Simulation Parameters

\begin{tabular}{ccc}
\hline parameters & description & value \\
\hline$R$ & Communication range & $300 \mathrm{~m}$ \\
$\beta$ & Vehicle arrival & $0.2,0.5,1$ \\
rate & Vehicle/second \\
$1 / \alpha$ & Average random interval & $1 \mathrm{~s}$ \\
$v_{\max }$ & length & \\
$v_{\min }$ & Maximum speed & $35 \mathrm{~m} / \mathrm{s}$ \\
$T_{s}$ & Minimum speed & $15 \mathrm{~m} / \mathrm{s}$ \\
\hline
\end{tabular}

In simulation, the vehicle speed changes from $15 \mathrm{~m} / \mathrm{s}$ to $35 \mathrm{~m} / \mathrm{s}$; according to DSRC [2], the vehicle communication radius is 300 meters. The simulation time for each experiment is 2000 seconds. Since the arrival rate $\beta$ obeys the Poisson distribution, the total number 
of vehicles entering the system is $2000 \times \beta$, and the specific simulation parameters are shown in Table 1. In the simulation process, due to the fluctuation in the initial phase of the system, the data of the initial 10 seconds and that of the last 10 seconds are ignored.

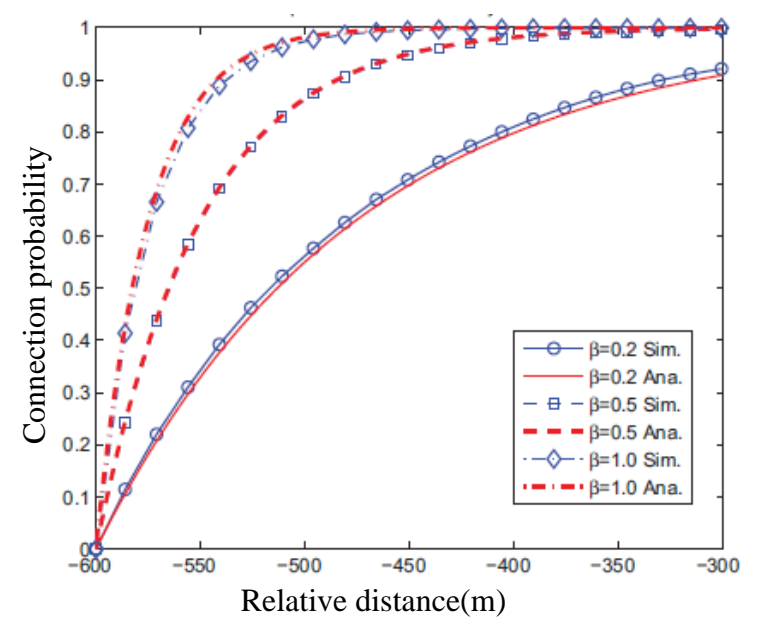

Figure 5. Changes in Two-Hop Connection Probability with the Arrival Rate $\beta$

Figure 5 shows the distribution of the two-hop connection probability. According to the figure, the two-hop connection probability of the reference node at the initial position 0 is exponentially distributed with the relative distance. The negative sign on the abscissa indicates that the destination node lags the reference node. With the reduction of the relative distance between the destination node and the reference node, the forwarding area in the middle will be expanded, thereby increasing the probability of emergence of intermediate nodes in the forwarding area and increasing the connection probability. Sim in Figure 5 represents the experimental simulation data, and Ana indicates the theoretical analysis data. Figure 5 shows that the analyzed performance is completely consistent with the experimental simulation results.

As is shown in Figure 5, when the relative distance between vehicles is up to -600 meters, the connection probability drops to 0 sharply, and this is because the region space for intermediate nodes is reduced. In addition, Figure 5 shows the changes in the connection probability with the arrival rate. According to the data in the graph, the connection probability decreases with $\beta$ changing from 1 to 0.5 and 0.2 . This is because the reduction of $\beta$ means that the number of vehicles per unit time is reduced, i.e. vehicle density decreases, resulting in reduction of intermediate forwarding vehicles and ultimately causing a decline in the rate of communication connection. When $\beta=0.2$, the connection probability located at -300 reached 0.9 . This also shows that when the vehicle density drops, it is very difficult to keep the vehicle network in the fully connected state. 


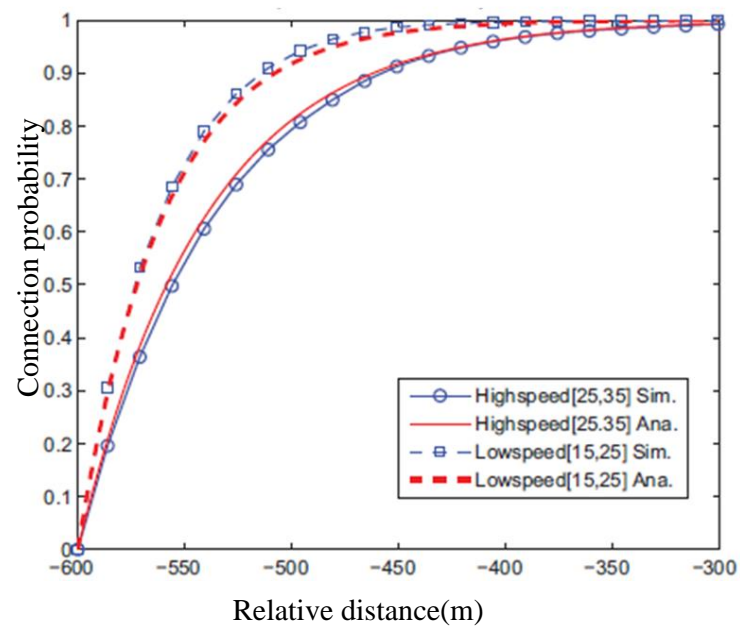

\section{Figure 6. Changes in Two-Hop Connection Probability with Vehicle Spped}

Figure 6 analyzes the effect of the speed variable on the two-hop connection probability. The arrival rate in Figure 6 is $0.5(\beta=0.5)$. The speed of the vehicle is divided into two groups: 25 to $35 \mathrm{~m} / \mathrm{s}$ for the high speed group; 15 to $25 \mathrm{~m} / \mathrm{s}$ for the low speed group. As is shown in Figure 6, in similar circumstances, the connection probability of the low speed group is higher than that of the high speed group, which is because when the vehicle speed is lower, the network topology is relatively stable, and it is more convenient for the intermediate forwarding nodes to forward the message. On the contrary, high speed driving aggravates the change of the network topology and increases the relative distance of vehicles, which leads to low connection probability. In addition, low speed driving can improve the traffic density and improve the life of the communication path.

\section{Conclusion}

For the multi-hop connection of VANETs, a new method is put forward to analyze the multi-hop based on a moving model and an analysis model is proposed to assess the availability of the two-hop path and to derive the vehicle emergence probability in the forwarding area and further the connection probability of indirect communication. When the distance between vehicles is greater than the communication radius, the connection is realized with the forwarding of the intermediate nodes, and the connection is quantified. The analysis results contribute to the routing decision and reducing the transmission delay caused by the multi-hop connection. In the future, how to use the connection probability to evaluate the life of multi-hop paths and improve the stability of the links will be studied.

\section{References}

[1] J. He, Y. Geng, F. Liu and C. Xu, "CC-KF: Enhanced TOA Performance in Multipath and NLOS Indoor Extreme Environment”, IEEE Sensor Journal, vol. 14, no. 11, (2014), pp. 3766-3774.

[2] S. Zhou, L. Mi, H. Chen and Y. Geng, "Building detection in Digital surface model", 2013 IEEE International Conference on Imaging Systems and Techniques (IST), (2012).

[3] N. Lu, C. Lu, Z. Yang and Y. Geng, "Modeling Framework for Mining Lifecycle Management", Journal of Networks, vol. 9, no. 3, (2014), pp. 719-725.

[4] Y. Geng and K. Pahlavan, "On the accuracy of rf and image processing based hybrid localization for wireless capsule endoscopy", IEEE Wireless Communications and Networking Conference (WCNC), (2015).

[5] G. Liu, Y. Geng and K. Pahlavan, "Effects of calibration RFID tags on performance of inertial navigation in indoor environment”, 2015 International Conference on Computing, Networking and Communications (ICNC), (2015). 
[6] Z. Lv, T. Yin, Y. Han, Y. Chen and G. Chen, "WebVR— — web virtual reality engine based on P2P network", Journal of Networks, vol. 6, no. 7, (2011), pp. 990-998.

[7] J. Yang, B. Chen, J. Zhou and Z. Lv, "A portable biomedical device for respiratory monitoring with a stable power source", Sensors, (2015).

[8] Z. Dongfang, "FusionFS: Toward supporting data-intensive scientific applications on extreme-scale high-performance computing systems." Big Data (Big Data), 2014 IEEE International Conference on. IEEE, (2014).

[9] S. Dang, J. Ju, D. Matthews, X. Feng and C. Zuo, "Efficient solar power heating system based on lenticular condensation", Information Science, Electronics and Electrical Engineering (ISEEE), 2014 International Conference on, (2014), pp. 26-28.

[10] X. Zhang, Y. Han, D. Hao and Z. Lv, “ARPPS : Augmented Reality Pipeline Prospect System”, 22th International Conference on Neural Information Processing (ICONIP 2015), In press, Istanbul, Turkey.

[11] J. Hu and Z. Gao, "Distinction immune genes of hepatitis-induced heptatocellular carcinoma[J]", Bioinformatics, vol. 28, no. 24, pp. 3191-3194.

[12] K. Wang, "Overcoming Hadoop Scaling Limitations through Distributed Task Execution"

[13] Z. Su, Xin Zhang, and Xinming Ou. "After we knew it: empirical study and modeling of costeffectiveness of exploiting prevalent known vulnerabilities across iaas cloud", Proceedings of the 9th ACM symposium on Information, computer and communications security, ACM, (2014).

[14] G. Bao, L. Mi, Y. Geng and K. Pahlavan, "A computer vision based speed estimation technique for localiz ing the wireless capsule endoscope inside small intestine", 36th Annual International Conference of the IEEE Engineering in Medicine and Biology Society (EMBC), (2014).

[15] W. Gu, Z. Lv and M. Hao, "Change detection method for remote sensing images based on an improved Markov random field", Multimedia Tools and Applications, (2016).

[16] Z. Lu, C. Esteve, J. Chirivella and P. Gagliardo, "A Game Based Assistive Tool for Rehabilitation of Dysphonic Patients", 3rd International Workshop on Virtual and Augmented Assistive Technology (VAAT) at IEEE Virtual Reality 2015 (VR2015), Arles, France, IEEE, (2015).

[17] Z. Chen, W. Huang and Z. Lv, "Uncorrelated Discriminant Sparse Preserving Projection Based Face Recognition Method", Multimedia Tools and Applications, (2016).

[18] Z. Lv, A. Halawani, S. Feng, H. Li and S. U. Rehman, "2014, Multimodal Hand and Foot Gesture Interaction for Handheld Devices", ACM Transactions on Multimedia Computing, Communications, and Applications (TOMM), vol. 11, no.1, Article 10, (2014), p. 19.

[19] K. Leng, W. Shi, J. Chen and Z. Lv, "Designing of a I-shaped less-than-truckload cross-dock, A simulation experiments study", International Journal of Bifurcation and Chaos, (2015).

[20] Y. Lin, J. Yang, Z. Lv, W. Wei and H. Song, "A Self-Assessment Stereo Capture Model”, Applicable to the Internet of Things, Sensors, (2015).

[21] J. He, Y. Geng and K. Pahlavan, "Toward Accurate Human Tracking: Modeling Time-of-Arrival for Wireless Wearable Sensors in Multipath Environment”, IEEE Sensor Journal, vol. 14, no. 11, (2014), pp. 3996-4006.

\section{Authors}

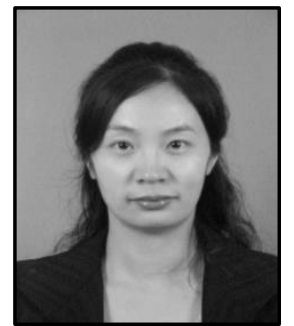

Zhang Tiandan received her M.S. degree in software engineering from University of Electronic Science and Technology of China. She is currently a lecturer in the Information Technology Department of Nantong Normal College. Her research interest is mainly in the area of Software Engineering, Computer Network. She has published several research papers in scholarly journals in the above research areas. 